\title{
Influencia del tamaño del grano en las propiedades mecánicas de los aceros inoxidables austeníticos
}

\author{
M.A. Martínez*, J. Ordieres", J. Botella**, R. Sánchez y R. Parra**
}

\begin{abstract}
Resumen En el presente trabajo se lleva a cabo un análisis de la influencia del tamaño del grano de aceros inoxidables austeníticos sobre algunas propiedades mecánicas de interés en estos materiales. Se han estudiado tanto aceros estables como metaestables para valorar las diferencias de comportamiento, considerando varias coladas de cada material para promediar los resultados y obtener un comportamiento más robusto de los estimadores. Se ha procedido a una preparación y adecuación de las probetas, previa a su ensayo en laboratorio. Se han observado los comportamientos habituales de modo que, a mayor número de tamaño de grano según norma ASTM, mayor límite elástico y resistencia a la tracción y menor alargamiento; llevándose a cabo estudios de detalle de valores de las tendencias de crecimiento, comportamiento comparado de estables y metaestables y valorándose cuidadosamente la influencia del níquel presente en el material. Finalmente se presentan las principales conclusiones y valoraciones, y un estudio estadístico de las propiedades mecánicas y de las distintas coladas para lograr una mejor predicción del comportamiento.
\end{abstract}

Palabras clave Tamaño de grano. Aceros inoxidables austeníticos estables. Aceros inoxidables austeníticos metaestables. Límite elástico. Resistencia a la tracción.

\section{Influence of grain size in mechanical properties of austenitic stainless steels}

Abstract

Keywords
The goal of this work is to study the influence of the grain size on mechanical properties of austenitic stainless steels. The study covers both, stable stainless steels and metastable steels. A relevant topic is to identify the differences in the behaviour of both types of steels. First of all, several steel taps are taken into account and by this way the average of results are derived. A sorted collection of them show its behaviour. Several rules are identified, like straight relationship between the number of grain size (ASTM) and the $0.2 \%$ proof stress and the tensile strength and inverse relationship with the elongation. Specific studies analyzing the relevance of nickel are performed. Finally main conclusions and valuations are presented and some statistic results of mechanics properties and steel taps are performed in order to get better prediction of their behaviour.

Grain size. Metastable steels. Stable steels. Elastic limit. Tensile strength.

\section{INTRODUCCIÓN}

Los aceros inoxidables austeníticos son ampliamente utilizados debido a su gran resistencia a la corrosión y trabajabilidad. Sin embargo, debido a su dureza relativamente alta es, a veces, difícil asegurar bastante formabilidad en el laminado y en el recocido. La producción e láminas de aceros inoxidables más blandas seguramente extenderá el uso de estos aceros. 
Apuntando a este objetivo, se han investigado los efectos de los elementos de aleación en las propiedades mecánicas de los aceros inoxidables austeníticos estables.

Los aceros inoxidables austeníticos metaestables experimentan una transformación de austenita a martensita cuando se deforman. Esta transformación más el endurecimiento por deformación de la austenita antes de la transformación y el efecto de fortalecimiento de la martensita formada durante la misma, contribuyen apreciablemente a su comportamiento plástico. También se han investigado los efectos de los elementos de aleación ${ }^{[1]}$ en las propiedades mecánicas, y en el fortalecimiento de la composición austenita-martensita en este tipo de aceros ${ }^{[2]}$.

Se ha pretendido por tanto, realizar un estudio sobre una serie de aceros inoxidables austeníticos tanto estables como metaestables, con la finalidad de ver la influencia de la composición química y de la estructura en las propiedades mecánicas de los mismos, ya que, con el tiempo se van produciendo cambios en los sistemas de producción y se obtienen aceros inoxidables que son aparentemente iguales a los que se fabricaban hace décadas, y, sin embargo, presentan diferencias que pareciendo sutiles llegan a ser sustancialmente importantes en algunos aspectos.
Así se tomarán materiales con composiciones químicas diferentes según diferentes grupos, y dentro de cada grupo se elegirán varios materiales de distintas coladas pero de composiciones químicas prácticamente invariables.

Se propone para cada grupo de austenitas, establecer la relación entre tamaño de grano y propiedades mecánicas ya que los granos, según su tamaño y proceso de formación, dan al metal características muy distintas. Cuanto mayor es el grano de que está constituido un metal, peores son, en general, sus propiedades mecánicas.

\section{MATERIALES Y PROCEDIMIENTO EXPERIMEN- TAL}

Se han tomado dos tipos de aceros inoxidables austeníticos estables, el AISI 316 y el 316L y dos tipos de aceros inoxidables austeníticos metaestables, del tipo AISI 304DDQ. De cada tipo de acero, se han tomado cuatro coladas ${ }^{[3]}$ diferentes con pequeñas variaciones de composición (tabla I).

Los aceros han sido laminados en caliente ${ }^{[4]}$ hasta un espesor de $2,5 \mathrm{~mm}$ y después han sufrido un recocido y un decapado. A continuación, con la cizalla, se cortaron siete tiras de cada tipo de $2,5 \mathrm{~mm}$ de espesor, $35 \mathrm{~mm}$ de anchura y $300 \mathrm{~mm}$ de longitud para laminar en frío ${ }^{[5]}$ con un

Tabla I. Composición de los aceros empleados, \% en masa

Table I. Composition of steels used, mass \%

\begin{tabular}{|c|c|c|c|c|c|c|c|c|c|c|c|c|c|c|c|c|c|c|c|}
\hline & Colada & $S$ & B & $C$ & $\mathrm{Cu}$ & $\mathrm{Cr}$ & $M n$ & Mo & $\mathrm{Ni}$ & $\mathbf{N}$ & Si & $\mathrm{Ti}$ & Al & Co & V & As & $\mathrm{Nb}$ & $\mathbf{P}$ & Sn \\
\hline & 1 & 0,003 & 5 & 0,028 & 0,299 & 17,432 & 1,025 & 0,315 & 8,489 & 0,0405 & 0,373 & 0,005 & 0,004 & 0,142 & 0,051 & 0,006 & 0,006 & 0,031 & 0,017 \\
\hline $\begin{array}{c}\text { Coladas AISI } \\
\text { 304DDQ }\end{array}$ & 2 & 0,004 & 6 & 0,025 & 0,357 & 17,654 & 1,049 & 0,362 & 8,738 & 0,0362 & 0,45 & 0,005 & 0,004 & 0,151 & 0,054 & 0,006 & 0,006 & 0,035 & 0,018 \\
\hline \multirow[t]{2}{*}{ Seleccionadas } & 3 & 0,005 & 5 & 0,031 & 0,285 & 17,411 & 0,975 & 0,353 & 8,597 & 0,0386 & 0,343 & 0,004 & 0,004 & 0,266 & 0,08 & 0,014 & 0,015 & 0,031 & 0,015 \\
\hline & 4 & 0,004 & 4 & 0,037 & 0,284 & 17,572 & 1,028 & 0,25 & 8,6 & 0,0388 & 0,377 & 0,005 & 0,004 & 0,169 & 0,066 & 0,011 & 0,006 & 0,03 & 0,014 \\
\hline & 5 & 0,004 & 4 & 0,025 & 0,351 & 17,818 & 1,017 & 0,272 & 9,204 & 0,0291 & 0,351 & 0,005 & 0,004 & 0,154 & 0,078 & 0,011 & 0,005 & 0,032 & 0,016 \\
\hline $\begin{array}{c}\text { Coladas AISI } \\
\text { 304DDQ }\end{array}$ & 6 & 0,003 & 6 & 0,038 & 0,424 & 17,707 & 1,124 & 0,438 & 9,343 & 0,0301 & 0,388 & 0,006 & 0,004 & 0,107 & 0,052 & 0,008 & 0,015 & 0,034 & 0,017 \\
\hline \multirow[t]{2}{*}{ Seleccionadas } & 7 & 0,004 & 18 & 0,033 & 0,298 & 17,845 & 0,995 & 0,301 & 9,149 & 0,0293 & 0,373 & 0,01 & 0,004 & 0,145 & 0,041 & 0,006 & 0,006 & 0,031 & 0,016 \\
\hline & 8 & 0,004 & 28 & 0,028 & 0,29 & 17,754 & 1,065 & 0,329 & 9,255 & 0,0317 & 0,392 & 0,02 & 0,004 & 0,146 & 0,05 & 0,006 & 0,004 & 0,032 & 0,013 \\
\hline & 9 & 0,003 & 21 & 0,042 & 0,428 & 17,238 & 1,231 & 2,195 & 10,851 & 0,0355 & 0,362 & 0,015 & 0,004 & 0,184 & 0,068 & 0,006 & 0,012 & 0,034 & 0,011 \\
\hline $\begin{array}{c}\text { Coladas AISI } \\
316\end{array}$ & 10 & 0,009 & 23 & 0,041 & 0,35 & 17,304 & 1,274 & 2,158 & 10,586 & 0,0403 & 0,465 & 0,012 & 0,005 & 0,152 & 0,066 & 0,007 & 0,008 & 0,033 & 0,01 \\
\hline \multirow[t]{3}{*}{ Seleccionadas } & 11 & 0,004 & 22 & 0,038 & 0,319 & 17,304 & 1,229 & 2,174 & 10,711 & 0,0451 & 0,302 & 0,024 & 0,005 & 0,146 & 0,078 & 0,008 & 0,01 & 0,028 & 0,011 \\
\hline & 12 & 0,004 & 21 & 0,04 & 0,468 & 17,101 & 1,3 & 2,131 & 10,72 & 0,0413 & 0,383 & 0,019 & 0,005 & 0,251 & 0,087 & 0,006 & 0,061 & 0,031 & 0,011 \\
\hline & 13 & 0,005 & 22 & 0,021 & 0,294 & 17,225 & 1,674 & 2,494 & 10,662 & 0,043 & 0,355 & 0,006 & 0,004 & 0,193 & 0,074 & 0,009 & 0,012 & 0,033 & 0,011 \\
\hline $\begin{array}{c}\text { Coladas AISI } \\
316 \mathrm{~L}\end{array}$ & 14 & 0,005 & 16 & 0,024 & 0,395 & 17,04 & 1,682 & 2,674 & 10,68 & 0,0519 & 0,471 & 0,015 & 0,005 & 0,153 & 0,076 & 0,006 & 0,017 & 0,026 & 0,008 \\
\hline \multirow[t]{2}{*}{ Seleccionadas } & 15 & 0,005 & 24 & 0,029 & 0,429 & 17,071 & 1,548 & 2,598 & 10,766 & 0,0392 & 0,377 & 0,015 & 0,005 & 0,217 & 0,087 & 0,006 & 0,035 & 0,029 & 0,011 \\
\hline & 16 & 0,004 & 17 & 0,023 & 0,393 & 17,093 & 1,657 & 2,755 & 10,844 & 0,0431 & 0,437 & 0,012 & 0,005 & 0,19 & 0,084 & 0,007 & 0,01 & 0,029 & 0,009 \\
\hline
\end{tabular}


laminador NORTON, hasta un espesor de $0,8 \mathrm{~mm}$. Así, se obtienen para cada tipo de acero 28 probetas, 7 de cada colada. Se procede a recocer estas 7 probetas de cada colada en un horno CARBOLITE RAPID HEATING con CONTROLADOR EUROTHERM, a $1.100{ }^{\circ} \mathrm{C}$ y a siete tiempos diferentes, $60 \mathrm{~s}, 70 \mathrm{~s}, 95 \mathrm{~s}, 165 \mathrm{~s}$, $200 \mathrm{~s}, 5 \mathrm{~min}$ y $12 \mathrm{~min}$, enfriando las tiras con una pistola de aire comprimido, con el fin de conseguir tamaños de grano diferentes, que mediremos por el método de interceptación, según la norma ASTM, con un microscopio LEICA Modelo REICHERT MeF 3 A con 100 aumentos.

Tras los recocidos y la toma de los tamaños de grano, se procede a medir la dureza Vickers con $300 \mathrm{~g}$ (HV300) de las citadas muestras, con el Durómetro VICKERS LECO V-100-C1, para lue- go mecanizarlas y obtener probetas de tracción normalizadas según la norma ASTM. Se ensayan, entonces, las probetas a tracción a temperatura ambiente $^{[6]}$ con la Máquina Universal ZWICK $1486,250 \mathrm{KN}$, y se obtienen los datos de las propiedades mecánicas restantes, a saber: límite elástico al 0,2\%, resistencia a la tracción y alargamiento.

\section{RESULTADOS}

Tras el procedimiento experimental indicado se recogen, en las tablas II y III, los resultados correspondientes en cuanto al límite elástico al 0,2\%, a la resistencia a la tracción, al alargamiento, a la dureza Vickers y al tamaño del grano de los aceros inoxidables austeníticos en estudio.

Tabla II. Propiedades mecánicas de los aceros AISI 304DDQ

Table II. Mechanicals properties of AISI 304DDQ steels

\begin{tabular}{|c|c|c|c|c|c|c|c|c|c|c|c|}
\hline Probeta & $\begin{array}{c}\mathrm{Rp}_{0,2} \\
\left(\mathrm{~N} / \mathrm{mm}^{2}\right)\end{array}$ & $\begin{array}{c}\mathrm{Rm} \\
\left(\mathrm{N} / \mathrm{mm}^{2}\right)\end{array}$ & $A(\%)$ & $\begin{array}{c}\text { HV } \\
300 \mathrm{~g}\end{array}$ & GS & Probeta & $\begin{array}{c}\mathrm{Rp}_{0,2} \\
\left(\mathrm{~N} / \mathrm{mm}^{2}\right)\end{array}$ & $\begin{array}{c}\mathrm{Rm} \\
\left(\mathrm{N} / \mathrm{mm}^{2}\right)\end{array}$ & $A(\%)$ & $\begin{array}{c}\mathrm{HV} \\
300 \mathrm{~g}\end{array}$ & GS \\
\hline $1 \mathrm{a}$ & 259 & 647 & 51,7 & 146 & 9,6 & $5 a$ & 249 & 620 & 49,3 & 145 & 10,3 \\
\hline $1 b$ & 248 & 641 & 55,8 & 144 & 8,2 & $5 b$ & 237 & 607 & 51,9 & 145 & 9,5 \\
\hline $1 c$ & 238 & 631 & 53,5 & 145 & 7,2 & $5 c$ & 227 & 601 & 49,5 & 137 & 7,7 \\
\hline $1 d$ & 223 & 629 & 58,3 & 135 & 6,6 & $5 d$ & 214 & 591 & 54 & 137 & 7,1 \\
\hline $1 e$ & 220 & 625 & 56,5 & 136 & 6,4 & $5 e$ & 210 & 585 & 55,6 & 134 & 7 \\
\hline $1 f$ & 212 & 615 & 56,4 & 140 & 6,1 & $5 f$ & 202 & 579 & 59,4 & 129 & 6,4 \\
\hline $1 \mathrm{~h}$ & 207 & 611 & 59,8 & 141 & 4,9 & $5 \mathrm{~h}$ & 195 & 566 & 56,6 & 130 & 5,5 \\
\hline $2 a$ & 267 & 641 & 49,2 & 149 & 9,9 & $6 a$ & 262 & 632 & 47,1 & 149 & 10,1 \\
\hline $2 b$ & 259 & 634 & 52,8 & 152 & 9,6 & $6 b$ & 251 & 624 & 49,8 & 139 & 9,7 \\
\hline $2 c$ & 248 & 640 & 55,5 & 144 & 7,9 & $6 c$ & 240 & 615 & 51,1 & 139 & 9,1 \\
\hline $2 d$ & 234 & 626 & 54,5 & 147 & 7,7 & $6 d$ & 227 & 606 & 51,5 & 146 & 7,6 \\
\hline $2 e$ & 226 & 609 & 56,1 & 141 & 7,1 & $6 e$ & 225 & 602 & 52 & 132 & 7,4 \\
\hline $2 f$ & 225 & 614 & 56,3 & 135 & 6,8 & $6 f$ & 223 & 600 & 52,4 & 133 & 6,8 \\
\hline $2 \mathrm{~h}$ & 217 & 610 & 55,8 & 137 & 5,2 & $6 \mathrm{~h}$ & 213 & 585 & 52,5 & 138 & 6,4 \\
\hline $3 a$ & 263 & 658 & 50,8 & 155 & 9,8 & $7 a$ & 241 & 619 & 53,1 & 148 & 9,8 \\
\hline $3 b$ & 253 & 651 & 52,6 & 150 & 9,8 & $7 b$ & 242 & 618 & 49,8 & 146 & 9,4 \\
\hline $3 c$ & 243 & 647 & 53,7 & 147 & 7,8 & $7 c$ & 227 & 613 & 54,2 & 145 & 8,3 \\
\hline $3 d$ & 224 & 634 & 54,9 & 149 & 6,8 & $7 d$ & 217 & 602 & 55,2 & 145 & 7,4 \\
\hline $3 e$ & 223 & 631 & 54,6 & 139 & 6,4 & $7 e$ & 215 & 597 & 54,6 & 137 & 7,1 \\
\hline $3 f$ & 215 & 626 & 55,6 & 140 & 6,0 & $7 f$ & 210 & 587 & 54,8 & 140 & 7,1 \\
\hline $3 \mathrm{~h}$ & 208 & 620 & 56,4 & 132 & 5,1 & $7 \mathrm{~h}$ & 208 & 590 & 54,2 & 138 & 6,3 \\
\hline $4 a$ & 288 & 665 & 47,1 & 166 & 15,2 & $8 a$ & 253 & 640 & 49,7 & 152 & 9,6 \\
\hline $4 b$ & 276 & 663 & 49,0 & 170 & 11,1 & $8 b$ & 241 & 630 & 48,7 & 154 & 9,7 \\
\hline $4 c$ & 259 & 654 & 52,8 & 155 & 10,0 & $8 c$ & 230 & 622 & 50,9 & 144 & 8,0 \\
\hline $4 d$ & 241 & 640 & 52,4 & 157 & 7,9 & $8 d$ & 220 & 615 & 52,8 & 142 & 7,6 \\
\hline $4 e$ & 238 & 640 & 53,6 & 149 & 7,7 & $8 e$ & 221 & 609 & 51,1 & 144 & 7,4 \\
\hline $4 f$ & 230 & 632 & 53,9 & 139 & 6,9 & $8 f$ & 222 & 611 & 53,8 & 144 & 7,0 \\
\hline $4 \mathrm{~h}$ & 223 & 624 & 55,2 & 142 & 6,2 & $8 \mathrm{~h}$ & 216 & 604 & 53,9 & 140 & 6,8 \\
\hline
\end{tabular}


Tabla III. Propiedades mecánicas del AISI 316 y del AISI 316L

Table III. Mechanicals properties of AISI 316 and AISI $316 \mathrm{~L}$

\begin{tabular}{|c|c|c|c|c|c|c|c|c|c|c|c|}
\hline Probeta & $\begin{array}{c}R p_{0,2} \\
\left(\mathrm{~N} / \mathrm{mm}^{2}\right)\end{array}$ & $\begin{array}{c}\mathrm{Rm} \\
\left(\mathrm{N} / \mathrm{mm}^{2}\right)\end{array}$ & $A(\%)$ & $\begin{array}{c}\mathrm{HV} \\
300 \mathrm{~g}\end{array}$ & GS & Probeta & $\begin{array}{c}R p_{0,2} \\
\left(\mathrm{~N} / \mathrm{mm}^{2}\right)\end{array}$ & $\begin{array}{c}\mathrm{Rm} \\
\left(\mathrm{N} / \mathrm{mm}^{2}\right)\end{array}$ & $A(\%)$ & $\begin{array}{c}\mathrm{HV} \\
300 \mathrm{~g}\end{array}$ & GS \\
\hline $9 a$ & 285 & 611 & 42,6 & 154 & 10,3 & $13 a$ & 300 & 632 & 43,4 & 160 & 10,5 \\
\hline $9 b$ & 281 & 609 & 45,1 & 149 & 9,9 & $13 b$ & 285 & 618 & 47,6 & 154 & 9,9 \\
\hline $9 c$ & 263 & 592 & 45,1 & 151 & 9,5 & $13 c$ & 273 & 611 & 45,7 & 157 & 9,3 \\
\hline $9 d$ & 256 & 590 & 47,0 & 144 & 9,3 & $13 d$ & 253 & 594 & 49 & 148 & 7,5 \\
\hline $9 e$ & 253 & 587 & 47,3 & 143 & 8,0 & $13 e$ & 247 & 587 & 48,4 & 142 & 7,3 \\
\hline $9 f$ & 248 & 579 & 47,0 & 135 & 7,9 & $13 f$ & 241 & 584 & 49,6 & 142 & 6,8 \\
\hline $9 \mathrm{~h}$ & 236 & 571 & 48,3 & 135 & 7,2 & $13 \mathrm{~h}$ & 228 & 570 & 49,4 & 138 & 5,2 \\
\hline $10 a$ & 319 & 646 & 41,5 & 176 & 15,5 & $14 a$ & 295 & 615 & 39,1 & 170 & 10,8 \\
\hline $10 \mathrm{~b}$ & 306 & 639 & 42,0 & 173 & 14,9 & $14 b$ & 276 & 603 & 43,7 & 163 & 9,9 \\
\hline $10 c$ & 281 & 617 & 46,9 & 161 & 10,5 & $14 c$ & 275 & 606 & 44,7 & 151 & 9,4 \\
\hline $10 d$ & 264 & 607 & 47,8 & 154 & 9,6 & $14 d$ & 265 & 597 & 45,9 & 153 & 7,6 \\
\hline $10 \mathrm{e}$ & 260 & 604 & 46,4 & 162 & 9,3 & $14 f$ & 261 & 596 & 46,1 & 153 & 7,6 \\
\hline $10 f$ & 255 & 601 & 48,1 & 149 & 7,6 & $14 \mathrm{e}$ & 255 & 588 & 45,5 & 147 & 7,5 \\
\hline $10 \mathrm{~h}$ & 243 & 582 & 47,1 & 141 & 7,1 & $14 \mathrm{~h}$ & 245 & 578 & 46 & 137 & 7,0 \\
\hline $11 a$ & 300 & 637 & 43,6 & 157 & 10,8 & $15 a$ & 293 & 613 & 38,9 & 156 & 10,4 \\
\hline $11 b$ & 288 & 623 & 41,0 & 159 & 10,2 & $15 b$ & 277 & 601 & 42,9 & 144 & 9,6 \\
\hline $11 \mathrm{c}$ & 276 & 618 & 44,7 & 155 & 9,7 & $15 c$ & 265 & 595 & 45,8 & 150 & 9,3 \\
\hline $11 d$ & 260 & 605 & 45,9 & 150 & 9,3 & $15 d$ & 258 & 585 & 46,7 & 143 & 7,9 \\
\hline $11 \mathrm{e}$ & 257 & 598 & 42,5 & 149 & 8,2 & $15 \mathrm{e}$ & 257 & 594 & 43,0 & 142 & 7,7 \\
\hline $11 \mathrm{f}$ & 253 & 598 & 49,6 & 149 & 7,8 & $15 f$ & 243 & 574 & 44,7 & 143 & 7,5 \\
\hline $11 \mathrm{~h}$ & 247 & 590 & 48,5 & 142 & 7,3 & $15 \mathrm{~h}$ & 236 & 573 & 45,1 & 140 & 7,0 \\
\hline $12 a$ & 292 & 620 & 43,3 & 156 & 10,4 & $16 a$ & 326 & 635 & 40,2 & 185 & 15,7 \\
\hline $12 b$ & 282 & 612 & 46,2 & 154 & 10,3 & $16 b$ & 297 & 617 & 40,1 & 171 & 14,4 \\
\hline $12 c$ & 269 & 599 & 45,9 & 153 & 9,8 & $16 c$ & 279 & 611 & 42,0 & 156 & 10,2 \\
\hline $12 d$ & 256 & 592 & 46,1 & 150 & 8,0 & $16 d$ & 264 & 593 & 43,0 & 153 & 9,7 \\
\hline $12 \mathrm{e}$ & 255 & 594 & 47,1 & 146 & 7,7 & $16 \mathrm{e}$ & 257 & 590 & 47,1 & 147 & 9,6 \\
\hline $12 f$ & 246 & 581 & 45,3 & 146 & 7,6 & $16 f$ & 251 & 586 & 47,1 & 140 & 7,8 \\
\hline $12 \mathrm{~h}$ & 238 & 568 & 46,0 & 136 & 6,9 & $16 \mathrm{~h}$ & 241 & 576 & 48,1 & 136 & 6,7 \\
\hline
\end{tabular}

Dentro de cada colada de los distintos aceros se designará como a, b, c, d, e, f, h a las muestras recocidas a los siete tiempos establecidos, esto es:
Para 60 s, a.
Para $70 \mathrm{~s}, \mathrm{~b}$.
Para 95 s, c.
Para 165 s, d.
Para 200 s, e.
Para $5 \mathrm{~min}, \mathrm{f}$.
Para $12 \mathrm{~min}, \mathrm{~h}$.

\section{DISCUSIÓN Y CONCLUSIONES}

A la vista de los resultados, se aprecia, como era de esperar, que conforme aumentamos el tiempo de recocido, el número del tamaño del grano según ASTM, disminuye; y conforme a esto, van variando las propiedades mecánicas de los distintos materiales.
En todos los aceros inoxidables austeníticos estudiados, tanto estables como metaestables, se observa la misma tónica: a mayor número de tamaño de grano (tamaño de grano más pequeño), mayores valores se obtienen de límite elástico al 0,2\%, de resistencia a la tracción ${ }^{[7]}$ y de dureza, y menores valores se obtienen en cuanto a los alargamientos.

Tomando como referencia un tamaño de grano igual a siete, observamos que los aceros metaestables tienen menores valores de límite elástico al $0,2 \%$ que los estables; mientras que, en cuanto a alargamientos y resistencias a la tracción tienen valores mayores, siendo las durezas muy similares. Esto puede atribuirse a las cantidades de manganeso, molibdeno y níquel ${ }^{[1]}$, que son mayores en los 


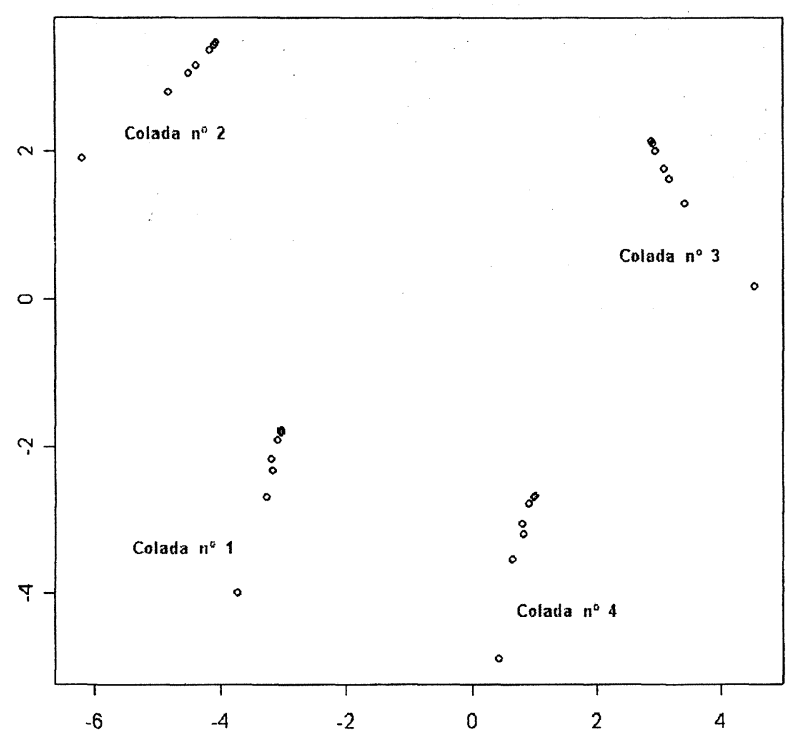

Figura 1. Proyección Sammon de las coladas correspondientes al AISI 304DDQ.

Figure 1. Proyection Sammon of tappings AISI 304 DDQ.

aceros inoxidables austeníticos estables. También influirá el tiempo de recocido, ya que en los aceros estables se consigue este tamaño de grano a mayor tiempo, $12 \mathrm{~min}$, que en los metaestables, que se consigue con $200 \mathrm{~s}$ de recocido.

Para menores tiempos de recocido, se observa prácticamente el mismo comportamiento descrito anteriormente entre los aceros inoxidables austeníticos estables y metaestables estudiados, aunque se aprecian resistencias a la tracción muy similares, en este caso, para los cuatro aceros.

De los dos aceros austeníticos metaestables del tipo AISI 304 DDQ, entre los que hay pequeñas variaciones notables de composición, se puede indicar que es el níquel ${ }^{[7]}$ el que más influye, de modo que el segundo de ellos, que tiene mayores proporciones de níquel, es el que tiene menores valores de sus propiedades mecánicas.

Analizando los resultados recogidos para los aceros inoxidables austeníticos estables ${ }^{[8]}$, el AISI 316 y el AISI 316L, se observa que, en cuanto a sus composiciones, los cambios más notables se aprecian en las cantidades de manganeso y molibdeno, ya que las cantidades de los restantes elementos presentan muy pocas variaciones de uno a otro. El AISI 316L, que es el que mayores proporciones tiene de los citados elementos, manganeso y molibdeno, es el que presenta menores valores del límite elástico al 0,2\%.

A la vista del elevado número de parámetros que definen la colada, se procedió a un estudio de Análisis de Componentes Principales (PCA) con variables centradas y normalizadas, incluyendo el tiempo de recocido, empleando la herramienta $\mathrm{R}{ }^{[9]}$. Este PCA revela que el $98 \%$ de la varianza es explicado por 5 vectores principales:

$$
\begin{gathered}
3,414990 \mathrm{e}+012,447841 \mathrm{e}+01 \quad 1,952974 \mathrm{e}+01 \\
1,092098 \mathrm{e}+01 \quad 1,092098 \mathrm{e}+01 \\
1,423257 \mathrm{e}-158,496003 \mathrm{e}-164,394419 \mathrm{e}-16 \\
1,586810 \mathrm{e}-171,001517 \mathrm{e}-17 \\
5,171095 \mathrm{e}-183,335930 \mathrm{e}-182,892357 \mathrm{e}-18 \\
1,624103 \mathrm{e}-181,359232 \mathrm{e}-18 \\
7,033654 \mathrm{e}-196,207045 \mathrm{e}-191,429825 \mathrm{e}-19 \\
0,000000 \mathrm{e}+000,000000 \mathrm{e}+00
\end{gathered}
$$

Esto permitiría no tener que medir los 20 parámetros de composición química sino sólo 5 de ellos para identificar la familia correctamente.

Adicionalmente, se ha procedido a efectuar una proyección MDS de Sammon para visualizar las clases que cada colada, incluyendo el tiempo de recocido, genera en un plano bidimensional con la condición de mantener las interdistancias en $\mathrm{R}^{21}$ lo más parecidas posible al espacio original.

El resultado puede visualizarse en el gráfico adjunto (Fig. 1).

\section{Agradecimientos}

Los autores agradecen a todo el personal de los laboratorios de la factoría de Acerinox en el Campo de Gibraltar, sin cuya ayuda hubiera sido imposible toda la realización práctica de este proyecto.

\section{REFERENCIAS}

[1] R. E. Trevisan, E. Braga y H.C. Fals, Rev. Metal. Madrid 38 (2002) 256-262.

[2] G.H. Eichelman, JR., y F.C. Hull, Trans. Am. Soc. Met. 45 (1952) 77-104.

[3] J. Botella, C. Merino, M.A. Heredia y J. Almagro, Rev. Metal. Madrid 35 (1999) 173-181.

[4] C. Camurri, M. López, J. Inostroza, M. Guzmán y J. A. JiMÉNEZ, Rev. Metal. Madrid 39 (2003) 35-40.

[5] G. De Diego, Ma L. Castaño y M. Hernández, Rev. Metal. Madrid 34 (1998) 407-420.

[6] A. Jirones, A. Mateo, L. Llanes y M. Anglada, Rev. Metal. Madrid 37 (2001) 150-154.

[7] K.A. Mohamed, A.A. Khedr, M.L. Mishreky y A.A. ABDul AzIM, Surf. Technol. 17 (1982) 11-18.

[8] N. Ohkubo, K. Miyakusu, Y. Uematsu y H. Kimura, ISiJ Int. 34 (1994) 764-772.

[9] http://www.r-project.org. Paquete estadístico de clasificación avanzada. 